\title{
The Mathematical Foundation of Symbolic Trajectory Evaluation
}

\author{
Ching-Tsun Chou* \\ Intel Corporation \\ 3600 Juliette Lane, SC12-401 \\ Santa Clara, CA 95052, U.S.A. \\ ctchou@mipos2.intel.com
}

\begin{abstract}
In this paper we elucidate the mathematical foundation underlying both the basic and the extended forms of symbolic trajectory evaluation (STE), with emphasis on the latter. The specific technical contributions we make to the theory of STE are threefold. First, we provide a satisfactory answer to the question: what does it mean for a circuit to satisfy a trajectory assertion? Second, we make the observation that STE is a form of data flow analysis and, as a corollary, propose a conceptually simple algorithm for extended STE. Third, we show that the theory of abstract interpretation based on Galois connections is the appropriate framework in which to understand STE.
\end{abstract}

\section{Introduction}

In BDD-based formal verification, symbolic trajectory evaluation (STE) [8,3] is the main alternative to symbolic model checking (SMC) [5]. Compared with SMC, STE has the advantage that it can be applied to very large circuits directly, without the need to abstract the circuits before verification. This is made possible by a pleasant property of STE: the number of BDD variables needed in an STE run depends only on the assertion being checked, not on the circuit under analysis. Thus one can use STE to verify a collection of assertions against the same circuit without having to invent a different abstraction of the circuit for each assertion, as one often has to do when doing SMC. On the other hand, what STE can verify is more restricted than what SMC can. In its basic form [8], STE can only verify assertions over bounded intervals of time, possibly iterated by non-nested loops. But in its extended form $[3]^{1}$, STE can verify assertions expressed as arbitrary state-transition graphs, thus enabling STE to verify any safety properties. As far as we know, STE has not been generalized to reason about liveness properties.

Unfortunately, STE seems to be much less well-known than SMC, certainly less than it deserves to be. Partly in the hope of generating more interests in STE, we elucidate in this paper the mathematical foundation underlying both

* The author is grateful to Pascalin Amagbegnon, John Mark Bouler, Pei-Hsin Ho, Marten van Hulst, Victor Konrad, Carl Seger, and the reviewers for comments and encouragements, and to his wife and children for love and tolerance.

1 According to Carl Seger, the basic ideas of extended (a.k.a. generalized) STE came out of an e-mail brainstorming session in 1994 among Derek Beatty, Randy Bryant, and Seger on a note written by Beatty, which unfortunately was never published. 
the basic [8] and the extended [3] forms of STE, with emphasis on the latter. The main mathematical theories used in this paper - data flow analysis $[4,6]$ and abstract interpretation $[1,7]$ - are not new. And it is quite possible that the basic ideas of this paper were already known at an intuitive level in the STE research community. But, to the best of our knowledge, these theories and ideas have never been brought together to form a coherent framework in which to understand (especially the extended form of) STE. The specific technical contributions we make to the theory of STE are threefold.

First, we clarify the semantics of STE by providing a satisfactory answer to the following question:

- What does it mean for a circuit to satisfy a trajectory assertion?

More precisely, we propose to define the satisfaction relation for extended STE [3], in which trajectory assertions can have arbitrary state-transition graphs, as a universally quantified generalization of the form of basic STE [8] in which trajectory assertions are bounded sequences of states. This is not how the satisfaction relation for extended STE was originally defined in [3], which uses a definition containing both universal and existential quantifiers. To justify our definition, we show that it guarantees that a circuit satisfies a trajectory assertion iff (if and only if) the set-theoretic STE algorithm returns a positive answer, and that this is not the case for the definition in [3]. Another advantage of our definition is that it does not require us to make the distinction of whether a trajectory assertion is "oblivious" (which basically means "deterministic"), whereas the definition in [3] does.

Second, we make the following observation:

- STE is a form of data flow analysis (DFA).

More precisely, we show that, when properly formulated, what an STE algorithm computes is exactly the solution of a data flow equation in the classic format [4, 6]. Though perhaps obvious in retrospect, this point seems to have never been noticed before. As a corollary of this DFA formulation, we propose a BDD-based, completely implicit algorithm for extended STE that is very easy to understand and, we hope, can lead to efficient implementations of STE. (Of course, this hope can be confirmed or disproved only through experimentation, which is beyond the scope of this paper.)

Third, we propose an appropriate framework in which to address the following question:

- How is the ternary model of circuits that STE algorithms use related to the ordinary boolean model of circuits?

Specifically, we show that the ternary model is an abstract interpretation in the classic sense [1,7] of the boolean model via a Galois connection [2,7]. We also point out a relationship between the two models (namely, the Galois connection should be a simulation from the boolean model to the ternary model) that seems to have never been articulated in the existing literature on STE $[8,3]$.

The rest of this paper is organized as follows. Section 2 presents STE from a set-theoretic viewpoint, in which circuits are modeled as functions operating 
on sets of boolean vectors. Section 3 presents STE from a lattice-theoretic viewpoint, in which circuits are modeled as functions operating on ternary vectors, which form a lattice. Section 4 presents the conceptually simple algorithm for extended STE mentioned above. The proofs of all theorems and the reviews of some mathematical machineries are relegated to the Appendices.

\section{Set-Theoretic STE}

Following [3], we start with set-theoretic STE, which manipulates sets of configurations of circuits. As will be seen, set-theoretic STE is impractical except on small circuits. But it provides an easy-to-understand semantic foundation by which STE can be related to symbolic model checking, which takes a set-theoretic view of circuits. Furthermore, the development of lattice-theoretic STE in the next section closely parallels that of set-theoretic STE.

\subsection{Set-Theoretic Models of Circuits}

Consider a digital circuit $M$ operating in discrete time. A configuration of $M$ is an assignment of "values" to "signals" in $M$, representing a snapshot of $M$ at a discrete point in time. In this section, exactly what "values" and "signals" are, is not important. All we need to assume is that the set of all configurations of $M$, denoted by $C$, is nonempty and finite.

Circuits as Relations. The conceptually simplest model of $M$ is a transition relation, $M_{\mathrm{Rel}} \subseteq C \times C$, where $\left(c, c^{\prime}\right) \in M_{\mathrm{Rel}}$ means that $M$ can in one step move from configuration $c$ to configuration $c^{\prime}$. Note that since $M$ cannot control its input signals, $M_{\mathrm{Rel}}$ is in general a relation rather than a function.

Circuits as Functions. The power set of $C$, denoted by $\mathcal{P}(C)$, can be viewed as the set of predicates on configurations, where $\cap, \cup$, and $\subseteq$ correspond to conjunction, disjunction, and implication, respectively. For any $Q \subseteq \mathcal{P}(C)$, we denote by $\cap Q$ and $\cup Q$ the intersection and union of all members of $Q$, respectively.

Using the relational image operation, the transition relation $M_{\text {Rel }}$ induces a predicate transformer $M_{\text {Fun }} \in \mathcal{P}(C) \rightarrow \mathcal{P}(C)$ in a natural way:

$$
M_{\text {Fun }}(p)=\left\{c^{\prime} \in C \mid \exists c \in p:\left(c, c^{\prime}\right) \in M_{\text {Rel }}\right\}
$$

for all $p \in \mathcal{P}(C)$. Intuitively, if $M$ is in one of the configurations in $p$, then in one step it must be in one of the configurations in $M_{\mathrm{Fun}}(p)$. It is easy to show from (1) that $M_{\text {Fun }}$ distributes over arbitrary union:

$$
M_{\mathrm{Fun}}(\cup Q)=\cup\left\{M_{\mathrm{Fun}}(q) \mid q \in Q\right\}
$$

for all $Q \subseteq \mathcal{P}(C)$. Conversely, for any $M_{\text {Fun }} \in \mathcal{P}(C) \rightarrow \mathcal{P}(C)$ that satisfies (2), the equivalence: $\left(c, c^{\prime}\right) \in M_{\mathrm{Rel}} \Leftrightarrow c^{\prime} \in M_{\mathrm{Fun}}(\{c\})$, where $c, c^{\prime} \in C$, defines a $M_{\mathrm{Rel}} \subseteq C \times C$ that satisfies (1). Thus there is no loss of information in going from $M_{\text {Rel }}$ to $M_{\text {Fun }}$ and vice versa.

In the remainder of this paper we will use the functional model of circuits exclusively and drop the subscript ${ }_{\text {Fun }}$. Note that it follows from distributivity (2) that $M\left(=M_{\text {Fun }}\right)$ both preserves $\emptyset$ (i.e., $\left.M(\emptyset)=\emptyset\right)$ and is monotonic (i.e., $p \subseteq q \Rightarrow M(p) \subseteq M(q)$ for all $p, q \in \mathcal{P}(C))$. 


\subsection{Set-Theoretic Trajectory Assertions}

From now on we focus on a fixed, but arbitrary, circuit $M \in \mathcal{P}(C) \rightarrow \mathcal{P}(C)$, where $C$ is nonempty and finite, such that (2) is true.

Definition of Trajectory Assertions. A trajectory assertion for $M$ is a quintuple $A=\left(S, s_{0}, R, \pi_{a}, \pi_{c}\right)$, where $S$ is a finite set of states, $s_{0} \in S$ is an initial state, $R \subseteq S \times S$ is a transition relation, and $\pi_{a} \in S \rightarrow \mathcal{P}(C)$ and $\pi_{c} \in S \rightarrow \mathcal{P}(C)$ label each state $s$ with an antecedent $\pi_{a}(s)$ and a consequent $\pi_{c}(s)$. Furthermore, we assume $\forall s \in S:\left(s, s_{0}\right) \notin R$, for the technical reason that in formulating data flow algorithms, it is convenient to have a unique source node whose flow value never needs changing. No generality is lost by making this assumption.

Satisfaction of a Trajectory Assertion by a Circuit. What does it mean for the circuit $M$ to satisfy the trajectory assertion $A=\left(S, s_{0}, R, \pi_{a}, \pi_{c}\right)$ ? Roughly speaking, it means that for every trajectory $\tau$ of $M$ and every run $\rho$ of $A$, as long as $\tau$ satisfies the antecedents in $\rho, \tau$ satisfies the consequents in $\rho$. To state this precisely, we have to introduce some terminologies. (Also, see Appendix A.1 for notations about sequences.)

A trajectory of $M$ is a nonempty sequence of configurations, $\tau \in C^{+}$, such that $\forall i \in \mathbf{N}: 0<i<|\tau| \Rightarrow \tau[i] \in M(\{\tau[i-1]\})$; the set of trajectories of $M$ is denoted by $\operatorname{Tr} a j(M)$. A run of $A$ is a nonempty sequence of states, $\rho \in S^{+}$, such that $\rho[0]=s_{0}$ and $\forall i \in \mathbf{N}: 0<i<|\rho| \Rightarrow(\rho[i-1], \rho[i]) \in R$; the set of runs of $A$ is denoted by $\operatorname{Runs}(A)$. Note that both $\operatorname{Traj}(M)$ and $\operatorname{Runs}(A)$ are prefix-closed. For any $\tau \in \operatorname{Traj}(M)$ and $\rho \in \operatorname{Runs}(A)$ such that $|\tau|=|\rho|$, we say $\tau$ a-satisfies (resp., $c$-satisfies) $\rho$, denoted by $\tau \models_{a} \rho$ (resp., $\tau \models_{c} \rho$ ), iff $\tau[i] \in \pi_{a}(\rho[i])$ (resp., $\left.\tau[i] \in \pi_{c}(\rho[i])\right)$ for each $i<|\tau|=|\rho|$. Finally, we say the circuit $M$ satisfies the trajectory assertion $A$, denoted by $M \models A$, iff:

$$
\forall \tau \in \operatorname{Traj}(M): \forall \rho \in \operatorname{Runs}(A):|\tau|=|\rho| \Rightarrow\left(\tau \models_{a} \rho \Rightarrow \tau \models_{c} \rho\right)
$$

Comparison with Another Definition of Satisfaction. It is instructive to compare (3) with the definition used in [3]:

$$
\begin{aligned}
& \forall \tau \in \operatorname{Traj}(M): \\
& \quad\left(\exists \rho \in \operatorname{Runs}(A):|\tau|=|\rho| \wedge \tau \models_{a} \rho \wedge \tau \models_{c} \rho\right) \vee \\
& \quad\left(\forall \tau^{\prime} \preceq \tau: \forall \rho^{\prime} \in \operatorname{Runs}(A):\left|\tau^{\prime}\right|=\left|\rho^{\prime}\right| \Rightarrow\left(\tau^{\prime} \models_{a} \rho^{\prime} \Rightarrow \tau^{\prime} \models_{c} \rho^{\prime}\right)\right)
\end{aligned}
$$

Note that (3) implies (4), because $\operatorname{Traj}(M)$ is prefix-closed. The converse is not true; but if its first disjunct were removed, (4) would indeed be equivalent to (3). That first disjunct, which contains an existential quantifier, makes (4) harder to implement than (3). Intuitively, the existential quantifier requires backtracking to implement. Formally, we will show in the next subsection that (3) holds iff the set-theoretic STE algorithm returns a positive answer, and that (4) lacks this nice property.

To get around this difficulty, [3] introduces the notion of oblivious trajectory assertions. A trajectory assertion $A=\left(S, s_{0}, R, \pi_{a}, \pi_{c}\right)$ is oblivious iff for any 
$s, s^{\prime}, s^{\prime \prime} \in S$ such that $\left(s, s^{\prime}\right) \in R$ and $\left(s, s^{\prime \prime}\right) \in R$, it must be the case that $\pi_{a}\left(s^{\prime}\right) \cap \pi_{a}\left(s^{\prime \prime}\right)=\emptyset$. Consequently, given any trajectory $\tau$, there is at most one run $\rho$ of $A$ such that $\tau a$-satisfies $\rho$. It is not hard to see that for an oblivious trajectory assertion, (4) implies (3), which then implies that (4) is equivalent to the set-theoretic STE algorithm returning a positive answer. With our definition (3), there is no need to introduce the notion of obliviousness.

\subsection{Set-Theoretic STE as DFA}

In this subsection we show that the checking of $M \models A$ can be formulated as a DFA problem $[4,6]$.

Define $F \in S \rightarrow(\mathcal{P}(C) \rightarrow \mathcal{P}(C))$ such that $F(s)(p)=M\left(\pi_{a}(s) \cap p\right)$ for all $s \in S$ and $p \in \mathcal{P}(C)$. It follows from (2) that for all $s \in S, F(s)$ preserves $\emptyset$ (i.e., $F(s)(\emptyset)=\emptyset)$, is monotonic (i.e., $p \subseteq q \Rightarrow F(s)(p) \subseteq F(s)(q)$ for all $p, q \in \mathcal{P}(C)$ ), and distributes over arbitrary union (i.e., $F(s)(\cup Q)=\cup\{F(s)(q) \mid q \in Q\}$ for all $Q \subseteq \mathcal{P}(C))$. Next, define $\mathcal{F} \in(S \rightarrow \mathcal{P}(C)) \rightarrow(S \rightarrow \mathcal{P}(C))$ such that:

$$
\mathcal{F}(\Phi)(s)=\text { if }\left(s=s_{0}\right) \text { then } C \text { else } \cup\left\{F\left(s^{\prime}\right)\left(\Phi\left(s^{\prime}\right)\right) \mid\left(s^{\prime}, s\right) \in R\right\}
$$

for all $\Phi \in S \rightarrow \mathcal{P}(C)$ and $s \in S$. Since $F(s)$ is monotonic for all $s \in S, \mathcal{F}$ is monotonic as well, where the function space $S \rightarrow \mathcal{P}(C)$ is ordered as follows: $\Phi \sqsubseteq \Phi^{\prime} \Leftrightarrow \forall s \in S: \Phi(s) \subseteq \Phi^{\prime}(s)$ for all $\Phi, \Phi^{\prime} \in S \rightarrow \mathcal{P}(C)$. Hence, by KnasterTarski Fixpoint Theorem [2], the fixpoint equation $\Phi=\mathcal{F}(\Phi)$ has a least solution $\Phi_{*} \in S \rightarrow \mathcal{P}(C)$. Furthermore, since both $S$ and $C$ are finite, $\Phi_{*}$ is the limit of the sequence $\left\langle\Phi_{n} \in S \rightarrow \mathcal{P}(C) \mid n \in \mathbf{N}\right\rangle$ defined by:

$$
\Phi_{n}=\text { if }(n=0) \text { then }(\lambda s \in S: \emptyset) \text { else } \mathcal{F}\left(\Phi_{n-1}\right)
$$

in the sense that there exists a sufficiently large $k \in \mathbf{N}$ such that $\Phi_{n}=\Phi_{*}$ for all $n \geq k$.

We say the circuit $M$ satisfies the trajectory assertion $A$ by set-theoretic $S T E$, denoted by $M \models_{\text {Set }} A$, iff $\forall s \in S: \Phi_{*}(s) \cap \pi_{a}(s) \subseteq \pi_{c}(s)$. Now we are ready to state our first main result:

Theorem 1. $M \models_{\text {Set }} A \Leftrightarrow M \models A$

Proof. See Appendix A.4.

Had we used the definition adopted in [3] for $M \models A$ (viz., (4) above), the $\Rightarrow$ direction of Theorem 1 would still be true (furthermore, obliviousness is not needed in this part of the proof; see Appendix A.4), but the $\Leftarrow$ direction would be false, as the following example shows. ${ }^{2}$ Consider a trivial circuit $M$ with only one signal whose value is either 0 or 1 (i.e., $C=\{0,1\}$ ); this signal is the output of a constant source 1 (i.e., $M(p)=\{1\}$ for $\emptyset \neq p \subseteq\{0,1\}$ ). Suppose that the trajectory assertion $A$ has only three states $S=\left\{s_{0}, s_{1}, s_{2}\right\}$ and two transitions $R=\left\{\left(s_{0}, s_{1}\right),\left(s_{0}, s_{2}\right)\right\}$ such that $\pi_{a}\left(s_{0}\right)=\pi_{a}\left(s_{1}\right)=\pi_{a}\left(s_{2}\right)=\pi_{c}\left(s_{0}\right)=\{0,1\}$ and $\pi_{c}\left(s_{1}\right)=\{1\}$ and $\pi_{c}\left(s_{2}\right)=\{0\}$. Then (4) is satisfied, because for any trajectory $\tau$ of $M$ with $|\tau|=2$, the run $\rho=\left\langle s_{0}, s_{1}\right\rangle$ satisfies both $\tau \models_{a} \rho$ and $\tau \models_{c} \rho$. But $M \not \forall_{\text {Set }} A$, since $\Phi_{*}\left(s_{2}\right)=\{1\}, \pi_{a}\left(s_{2}\right)=\{0,1\}$, but $\pi_{c}\left(s_{2}\right)=\{0\}$.

2 Space limitations prevent us from including state-transition diagrams for this and subsequent examples, but the reader should have no trouble drawing his own. 


\section{Lattice-Theoretic STE}

The definition (5) of the sequence $\left\langle\Phi_{n} \mid n \in \mathbf{N}\right\rangle$ above yields a simple method for computing the least fixpoint solution $\Phi_{*}$ : just compute $\Phi_{0}, \Phi_{1}, \Phi_{2}, \ldots$ one by one until a fixpoint, which must be $\Phi_{*}$, is reached. Then, by Theorem $1, M \models A$ can be checked by checking $M \models_{\text {Set }} A$. Since all objects involved are finite, this scheme for checking $M \models A$, which we call the set-theoretic STE algorithm, is clearly effective.

Unfortunately, the set-theoretic STE algorithm is not practical except for small circuits. For, if the circuit $M$ has $m$ boolean signals, then its set of configurations is $\mathbf{B}^{m}$, where $\mathbf{B}=\{0,1\}$ is the set of boolean values. Even with state-of-the-art BDD technologies, manipulating subsets of $\mathbf{B}^{m}$ is impractical for even moderately large $m$, say several hundred signals. But interesting circuits in the real world often contain thousands of (if not more!) signals, for which set-theoretic STE is powerless.

In this section we will describe what may be regarded as the key insight of the STE paradigm. Namely, instead of manipulating subsets of $\mathbf{B}^{m}$ directly, we approximate them with ternary vectors, whose sizes are only linear in $m$. But, to compensate for possible loss of information in the approximation process, we may have to complicate the trajectory assertion, or use a family of trajectory assertions, or both. Yet, in both cases, the number of BDD variables depends only on the trajectory assertion(s) and not on the circuit under analysis. This makes it possible to do STE on very large circuits without first abstracting them.

We will use many concepts and notations from the theory of partial orders and lattices [2]. In particular, the notions of complete lattices and Galois connections are reviewed in Appendices A.2 and A.3, respectively.

\subsection{Lattice-Theoretic Models of Circuits}

Recall that $M \in \mathcal{P}(C) \rightarrow \mathcal{P}(C)$ represents a circuit such that (2) is true, and that the set $C$ of configurations of $M$ is nonempty and finite. What exactly $C$ is, is not important until Section 4 .

Let $(\hat{P}, \sqsubseteq)$ be a finite complete lattice of abstract predicates such that there is a Galois connection $\ll \subseteq \mathcal{P}(C) \times \hat{P}$. An abstract predicate transformer $\hat{M} \in \hat{P} \rightarrow$ $\hat{P}$ is an abstract interpretation $[1,7]$ of $M \in \mathcal{P}(C) \rightarrow \mathcal{P}(C)$ iff $\hat{M}$ preserves bottom (i.e., $\hat{M}(\hat{\perp})=\hat{\perp}), \hat{M}$ is monotonic (i.e., $\hat{p} \sqsubseteq \hat{q} \Rightarrow \hat{M}(\hat{p}) \sqsubseteq \hat{M}(\hat{q})$ for all $\hat{p}, \hat{q} \in \hat{P})$, and $\ll$ is a simulation relation from $\mathcal{P}(C)$ to $\hat{P}$ (i.e., $p \ll \hat{p} \Rightarrow M(p) \ll \hat{M}(\hat{p})$ for all $p \in \mathcal{P}(C)$ and $\hat{p} \in \hat{P})$. That the Galois connection $\ll$ is a simulation relation can also be stated in terms of its abstraction function $\alpha: \mathcal{P}(C) \rightarrow \hat{P}$ (viz., $\alpha(M(p)) \sqsubseteq \hat{M}(\alpha(p))$ for all $p \in \mathcal{P}(C))$ or in terms of its concretization function $\gamma: \hat{P} \rightarrow \mathcal{P}(C)$ (viz., $M(\gamma(\hat{p})) \subseteq \gamma(\hat{M}(\hat{p}))$ for all $\hat{p} \in \hat{P})$. Although this notion of simulation is standard in the literature on abstract interpretation $[1,7]$, it seems to have never been used in the literature on STE $[8,3]$. It would be interesting to check whether actual implementations of STE satisfy this condition.

We do not require of $\hat{M}$ the counterpart of $(2): \hat{M}(\sqcup \hat{Q})=\sqcup\{\hat{M}(\hat{q}) \mid \hat{q} \in \hat{Q}\}$, because it is not true in general. For example, suppose $\hat{M}$ abstracts a unit-delay 
two-input AND-gate using ternary values. Then it is reasonable to require:

$$
\begin{aligned}
\hat{M}(\langle 0,1, \mathrm{X}\rangle \sqcup\langle 1,0, \mathrm{X}\rangle)=\hat{M}(\langle\mathrm{X}, \mathrm{X}, \mathrm{X}\rangle) & =\langle\mathrm{X}, \mathrm{X}, \mathrm{X}\rangle \\
\hat{M}(\langle 0,1, \mathrm{X}\rangle) \sqcup \hat{M}(\langle 1,0, \mathrm{X}\rangle)=\langle\mathrm{X}, \mathrm{X}, 0\rangle \sqcup\langle\mathrm{X}, \mathrm{X}, 0\rangle & =\langle\mathrm{X}, \mathrm{X}, 0\rangle
\end{aligned}
$$

where the first two vector components correspond to the two inputs and the last the output. Intuitively, the join operation $\langle 0,1, \mathrm{X}\rangle \sqcup\langle 1,0, \mathrm{X}\rangle=\langle\mathrm{X}, \mathrm{X}, \mathrm{X}\rangle$ throws away the information that one of the inputs is 0 , so $\hat{M}$ can no longer assign 0 to the output. Note, however, that the inequality $\hat{M}(\sqcup \hat{Q}) \sqsupseteq \sqcup\{\hat{M}(\hat{q}) \mid \hat{q} \in \hat{Q}\}$ does hold, since it is implied by the monotonicity of $\hat{M}$.

\subsection{Lattice-Theoretic Trajectory Assertions}

A trajectory assertion for $\hat{M}$ is a quintuple $\hat{A}=\left(S, s_{0}, R, \hat{\pi}_{a}, \hat{\pi}_{c}\right)$, where the assumptions on $S, s_{0}$, and $R$ are the same as in Section 2.2, and $\hat{\pi}_{a} \in S \rightarrow \hat{P}$ and $\hat{\pi}_{c} \in S \rightarrow \hat{P}$ are the antecedent and consequent labeling functions, respectively. Define $\gamma(\hat{A})=\left(S, s_{0}, R, \gamma\left(\hat{\pi}_{a}\right), \gamma\left(\hat{\pi}_{c}\right)\right)$, where $\gamma\left(\hat{\pi}_{a}\right)=\lambda s \in S: \gamma\left(\hat{\pi}_{a}(s)\right)$ and $\gamma\left(\hat{\pi}_{c}\right)=\lambda s \in S: \gamma\left(\hat{\pi}_{c}(s)\right)$. Note that $\gamma(\hat{A})$ is a trajectory assertion for $M$.

\subsection{Lattice-Theoretic STE as DFA}

Define $\hat{F} \in S \rightarrow(\hat{P} \rightarrow \hat{P})$ such that $\hat{F}(s)(\hat{p})=\hat{M}\left(\hat{\pi}_{a}(s) \sqcap \hat{p}\right)$ for all $s \in S$ and $\hat{p} \in \hat{P}$. For any $s \in S$, since $\hat{M}$ preserves bottom and is monotonic, $\hat{F}(s)$ also preserves bottom (i.e., $\hat{F}(s)(\hat{\perp})=\hat{\perp})$ and is monotonic (i.e., $\hat{p} \sqsubseteq \hat{q} \Rightarrow \hat{F}(s)(\hat{p}) \sqsubseteq$ $\hat{F}(s)(\hat{q})$ for all $\hat{p}, \hat{q} \in \hat{P})$. Next, define $\hat{\mathcal{F}} \in(S \rightarrow \hat{P}) \rightarrow(S \rightarrow \hat{P})$ such that:

$$
\hat{\mathcal{F}}(\hat{\Phi})(s)=\operatorname{if}\left(s=s_{0}\right) \text { then } \hat{\top} \text { else } \sqcup\left\{\hat{F}\left(s^{\prime}\right)\left(\hat{\Phi}\left(s^{\prime}\right)\right) \mid\left(s^{\prime}, s\right) \in R\right\}
$$

for all $\hat{\Phi} \in S \rightarrow \hat{P}$ and $s \in S$. Since $\hat{F}(s)$ is monotonic for all $s \in S, \hat{\mathcal{F}}$ is monotonic as well, where the function space $S \rightarrow \hat{P}$ is ordered as follows: $\hat{\Phi} \sqsubseteq \hat{\Phi}^{\prime} \Leftrightarrow \forall s \in$ $S: \hat{\Phi}(s) \sqsubseteq \hat{\Phi}^{\prime}(s)$ for all $\hat{\Phi}, \hat{\Phi}^{\prime} \in S \rightarrow \hat{P}$. Hence, by Knaster-Tarski Fixpoint Theorem [2], the fixpoint equation $\hat{\Phi}=\hat{\mathcal{F}}(\hat{\Phi})$ has a least solution $\hat{\Phi}_{*} \in S \rightarrow \hat{P}$. Furthermore, since both $S$ and $\hat{P}$ are finite, $\hat{\Phi}_{*}$ is the limit of the sequence $\left\langle\hat{\Phi}_{n} \in S \rightarrow \hat{P} \mid n \in \mathbf{N}\right\rangle$ defined by:

$$
\hat{\Phi}_{n}=\text { if }(n=0) \text { then }(\lambda s \in S: \hat{\perp}) \text { else } \hat{\mathcal{F}}\left(\hat{\Phi}_{n-1}\right)
$$

in the sense that there exists a sufficiently large $k \in \mathbf{N}$ such that $\hat{\Phi}_{n}=\hat{\Phi}_{*}$ for all $n \geq k$.

We say the abstract circuit $\hat{M}$ satisfies the abstract trajectory assertion $\hat{A}$ by lattice-theoretic $S T E$, denoted by $\hat{M} \models_{\text {Lat }} \hat{A}$, iff $\forall s \in S: \hat{\Phi}_{*}(s) \sqcap \hat{\pi}_{a}(s) \sqsubseteq \hat{\pi}_{c}(s)$. Now we are ready to state our second main result:

Theorem 2. If $\hat{M}$ is an abstract interpretation of $M$, then:

$$
\hat{M} \models_{\text {Lat }} \hat{A} \Rightarrow M=_{\text {Set }} \gamma(\hat{A})
$$

Proof. See Appendix A.5. 
The converse of Theorem 2 is not true. For example, consider a circuit with five signals $\left\langle i_{1}, i_{2}, j_{1}, j_{2}, o\right\rangle$, where $j_{1}$ (resp., $j_{2}$ ) is $i_{1}\left(i_{2}\right)$ delayed by one unit of time and $o$ is the unit-delayed AND of $j_{1}$ and $j_{2}$. Suppose that the trajectory assertion has five states $\left\{s_{0}, s_{1}, s_{1}^{\prime}, s_{2}, s_{3}\right\}$ and five transitions $\left\{\left(s_{0}, s_{1}\right),\left(s_{0}, s_{1}^{\prime}\right),\left(s_{1}, s_{2}\right)\right.$, $\left.\left(s_{1}^{\prime}, s_{2}\right),\left(s_{2}, s_{3}\right)\right\}$ and the labeling: $\pi_{a}\left(s_{1}\right)=\langle 0,1, \mathrm{X}, \mathbf{X}, \mathbf{X}\rangle, \pi_{a}\left(s_{1}^{\prime}\right)=\langle 1,0, \mathbf{X}, \mathbf{X}, \mathbf{X}\rangle$, $\pi_{c}\left(s_{3}\right)=\langle\mathbf{X}, \mathbf{X}, \mathbf{X}, \mathbf{X}, 0\rangle$; all other labels are $\langle\mathbf{X}, \mathbf{X}, \mathbf{X}, \mathbf{X}, \mathbf{X}\rangle$. Intuitively, the antecedent at $s_{1}$ (resp., $s_{1}^{\prime}$ ) assumes that $i_{1}=0$ and $i_{2}=1$ (resp., $i_{1}=1$ and $i_{2}=0$ ) at time 1 , and the consequent at $s_{3}$ checks that at time $3, o=0$ regardless of which assumption was used. It is easy to verify that for this example, $M \models_{\text {set }} \gamma(\hat{A})$ but $\hat{M} \not \nvdash_{\text {Lat }} \hat{A}$. And the reason is simple: at time 2 , when the information from $s_{1}$ and $s_{1}^{\prime}$ is merged at $s_{2}$, we have:

$$
\{\langle 0,1\rangle\} \cup\{\langle 1,0\rangle\}=\{\langle 0,1\rangle,\langle 1,0\rangle\} \quad \text { but } \quad\langle 0,1\rangle \sqcup\langle 1,0\rangle=\langle\mathrm{X}, \mathrm{X}\rangle
$$

the latter of which loses information. Clearly, this merge could be avoided by duplicating $s_{2}$ and $s_{3}$, so that there is a separate copy of them to deal with each of the assumptions $\pi_{a}\left(s_{1}\right)$ and $\pi_{a}\left(s_{1}^{\prime}\right)$. But then the number of states in the trajectory assertion increases. This kind of trade-offs between complexity and precision is typical of STE.

\section{An Implicit Algorithm for Lattice-Theoretic STE}

Up to this point, except in a few examples, we have not needed to specify what exactly the set $C$ of configurations is, except that $C$ should be nonempty and finite. This makes our theory more general. But in order to have a BDD-based implementation, we have to make up our mind now as to what $C$ is. Thus, in this section, we shall assume that $C=\mathbf{B}^{m}$ for some $m \in \mathbf{N}$. In other words, $M$ is a boolean circuit with $m$ signals. Furthermore, we assume that the abstract circuit $\hat{M}$ operates on ternary vectors, i.e., $\hat{P}=\mathbf{T}_{\perp}^{m}$. How sets of boolean vectors can be approximated by ternary vectors is explained in Appendix A.3.

Similar to the set-theoretic case, the definition (6) of the sequence $\left\langle\hat{\Phi}_{n}\right| n \in$ N) yields a simple algorithm for checking $\hat{M} \models_{\text {Lat }} \hat{A}$ : compute $\hat{\Phi}_{0}, \hat{\Phi}_{1}, \hat{\Phi}_{2}, \ldots$ one by one until a fixpoint, which must be $\hat{\Phi}_{*}$, is reached; then check $\left.\hat{M}\right|_{\text {Lat }} \hat{A}$ using its definition. Note that since the converse of Theorem 2 is not true, this algorithm, which we call the lattice-theoretic STE algorithm, can give falsely negative answers (i.e., when $\hat{M} \models_{\text {Lat }} \hat{A}$ but $\left.M \models \gamma(\hat{A})\right)$. But, by virtue of Theorems 1 and 2, it can never produce falsely positive answers (i.e., $\hat{M}==_{\text {Lat }} \hat{A}$ does imply $M \models \gamma(\hat{A}))$. We now argue that the lattice-theoretic STE algorithm can be implemented using BDDs in a straightforward manner.

First, notice that every ternaray value $t \in \mathbf{T}$ can be encoded with two boolean values: $B_{0}(t)=(0 \sqsubseteq t)$ and $B_{1}(t)=(1 \sqsubseteq t)$. With this encoding, join and meet are implemented by: $B_{i}\left(t \sqcup t^{\prime}\right)=B_{i}(t) \vee B_{i}\left(t^{\prime}\right)$ and $B_{i}\left(t \sqcap t^{\prime}\right)=B_{i}(t) \wedge B_{i}\left(t^{\prime}\right)$, where $i \in \mathbf{B}$. For any $m \in \mathbf{N}$, this encoding and the associated join and meet operations can be extended component-wise to $\mathbf{T}_{\perp}^{m}$. Note that $\perp$ has multiple encodings (viz., all $m$-tuples of boolean pairs in which at least one of the pairs is such that $B_{0}=B_{1}=0$ ). 
Without loss of generality, suppose the state space $S$ of the trajectory assertion is $\mathbf{B}^{k}$, for some $k \in \mathbf{N}$. With the above encoding of ternary values, the objects manipulated by the lattice-theoretic STE algorithm have the following types: $R \in \mathbf{B}^{k} \times \mathbf{B}^{k} \rightarrow \mathbf{B}$ and $\hat{\pi}_{a}, \hat{\pi}_{c}, \hat{\Phi}_{n}, \hat{\Phi}_{*} \in \mathbf{B}^{k} \rightarrow(\mathbf{B} \times \mathbf{B})^{m}$, for all $n \in \mathbf{N}$. It is not hard to see that these objects can all be represented by BDDs on at most $2 k$ variables, and that $\hat{\mathcal{F}}$ and the checking of $\hat{M}==_{\text {Lat }} \hat{A}$ can be implemented by BDD operations on these BDDs, provided that the output of the abstract circuit $\hat{M} \in \mathbf{T}_{\perp}^{m} \rightarrow \mathbf{T}_{\perp}^{m}$ for any given input can be computed without ever having to represent $\hat{M}$ itself as BDDs (which would require $2 m$ variables). Real-world STE implementations amply demonstrate that this proviso is practical.

We emphasize again that the maximum number of boolean variables needed by our algorithm, $2 k$, depends only on the trajectory assertion and not on the circuit. Of course, this independence is somewhat illusory, since the possible loss of information in the approximation by ternary vectors may necessitate more complex state-transition structure in the trajectory assertion, which would increase $k$. Furthermore, note that our formulation so far has been "unparameterized" in the sense that the antecedents and consequents are simple ternary vectors without parameters. In fact, they can be parameterized by boolean variables, so that a single run of the parameterized algorithm is equivalent to multiple runs of the unparameterized algorithm, one for each truth assignment to the boolean parameters. Needless to say, such parameters increase further the total number of boolean variables.

Despite its simplicity, the STE algorithm outlined above does not seem to have ever been implemented. Can it be as efficient in practice as, or even more so than, current implementations of extended STE? We do not know, but it would be interesting to find out.

\section{A Appendices}

\section{A.1 Sequences}

Let $\mathbf{N}=\{0,1,2, \cdots\}$ be the set of natural numbers. For any set $V$ and any $n \in \mathbf{N}, V^{n}$ (resp., $V^{+}$and $V^{*}$ ) denotes the set of all finite sequences of length $n$ (resp., positive and nonnegative lengths) over $V$. Let $\sigma, \tau \in V^{*}$. The length of $\sigma$ is denoted by $|\sigma|$, the concatenation of $\sigma$ followed by $\tau$ by $\sigma^{\frown} \tau$, and $\sigma$ being a prefix of $\tau$ by $\sigma \preceq \tau$. A set $S \subseteq V^{*}$ is prefix-closed iff $\sigma \in S$ and $\tau \preceq \sigma$ imply $\tau \in S$. For any $i \in \mathbf{N}$ with $0 \leq i<|\sigma|$, the $i$-th element of $\sigma$ is denoted by $\sigma[i]$. (Note that we index sequence elements starting from 0 instead of 1.) The last element of $\sigma$ is denoted by last $(\sigma)$, i.e., $\operatorname{last}(\sigma)=\sigma[|\sigma|-1]$. The empty sequence (i.e., the sequence whose length is 0 ) is denoted by \langle\rangle . A sequence consisting of elements $v_{0}, v_{1}, v_{2}, \cdots, v_{n-1} \in V$ (in that order) is written as $\left\langle v_{0}, v_{1}, v_{2}, \cdots, v_{n-1}\right\rangle$. We use the terms "sequences" and "vectors" interchangeably; the elements of vectors are sometimes referred to as "components".

\section{A.2 Complete Lattices}

A complete lattice is a poset $(P, \sqsubseteq)$ in which the meet and join of elements of any subset $Q \subseteq P$, denoted by $\sqcap Q$ and $\sqcup Q$ respectively, always exist. Intuitively, we think of the elements of a complete lattice as "predicates", so that $\sqcap$, $\sqcup$, and $\sqsubseteq$ corresponds to "conjunction", "disjunction", and "implication", respectively. 
For any set $V$, its power set $\mathcal{P}(V)$, ordered by set inclusion $\subseteq$, forms a complete lattice. Here $\sqcap, \sqcup$, and $\sqsubseteq$ are $\cap, \cup$, and $\subseteq$, respectively.

Let $\mathbf{T}=\{0,1, \mathbf{X}\}$ be the set of ternary values, where $\mathbf{X}$ denotes an unknown value. Intuitively, $X$ signifies a lack of information: it could be 0 , it could be 1 ; we simply don't know. We partially order $\mathbf{T}$ as follows: ${ }^{3} 0 \sqsubseteq \mathbf{X}$ and $1 \sqsubseteq \mathbf{X}$. For any $m \in \mathbf{N}$, this order on $\mathbf{T}$ is extended component-wise to $\mathbf{T}^{m}$. But $\left(\mathbf{T}^{m}, \sqsubseteq\right)$ is not a complete lattice, because it lacks a bottom. We fix this by introducing a special bottom element, $\perp$, such that $\perp \sqsubseteq t$ and $\perp \neq t$ for all $t \in \mathbf{T}^{m}$. Now $\mathbf{T}_{\perp}^{m}=\mathbf{T}^{m} \cup\{\perp\}$, ordered by $\sqsubseteq$, is indeed a complete lattice. We denote the top element $\langle\mathbf{X}, \cdots, \mathbf{X}\rangle$ of $\mathbf{T}_{\perp}^{m}$ by $\top$.

\section{A.3 Galois Connections}

Galois Connections as Relations. Let $\left(P^{b}, \sqsubseteq^{b}\right)$ and $\left(P^{\sharp}, \sqsubseteq^{\sharp}\right)$ be complete lattices of "concrete predicates" and "abstract predicates", respectively. (Below we will drop b and ${ }^{\sharp}$ from $\sqsubseteq^{b}$ and $\Xi^{\sharp}$ and the meet and join operations they induce, since they will always be clear from the context.) A Galois connection $[2,7]$ from $P^{b}$ to $P^{\sharp}$ is a binary

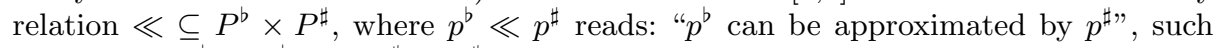
that for all $\bar{Q}^{b} \subseteq P^{b}$ and $Q^{\sharp} \subseteq P^{\sharp}$ :

$$
Q^{b} \ll Q^{\sharp} \Leftrightarrow \sqcup Q^{b} \ll \sqcap Q^{\sharp}
$$

where we define: $Q^{b} \ll Q^{\sharp} \Leftrightarrow \forall p^{b} \in Q^{b}: \forall p^{\sharp} \in Q^{\sharp}: p^{b} \ll p^{\sharp}$. Intuitively, (7) says that the approximation relation $\ll$ is an extension of the partial orders inside $P^{b}$ and $P^{\sharp}$ to between $P^{b}$ and $P^{\sharp}$.

Galois Connections as Functions. The usual definitions of Galois connections in the literature $[2,7]$ are in terms of an abstraction function $\alpha: P^{b} \rightarrow P^{\sharp}$ and a concretization function $\gamma: P^{\sharp} \rightarrow P^{b}$, which in our framework can be derived from $\ll$ as follows:

$$
\alpha\left(p^{b}\right)=\sqcap\left\{p^{\sharp} \in P^{\sharp} \mid p^{b} \ll p^{\sharp}\right\} \quad \gamma\left(p^{\sharp}\right)=\sqcup\left\{p^{b} \in P^{b} \mid p^{b} \ll p^{\sharp}\right\}
$$

for all $p^{b} \in P^{b}$ and $p^{\sharp} \in P^{\sharp}$. Intuitively, $\alpha\left(p^{b}\right)$ (resp., $\left.\gamma\left(p^{\sharp}\right)\right)$ is the most precise approximation of $p^{b}\left(p^{\sharp}\right)$ in $P^{\sharp}\left(P^{b}\right)$. Conversely, the relation $\ll$ can be derived from $\alpha$ or $\gamma$ as follows:

$$
p^{b} \ll p^{\sharp} \Leftrightarrow \alpha\left(p^{b}\right) \sqsubseteq p^{\sharp} \quad \quad p^{b} \ll p^{\sharp} \Leftrightarrow p^{b} \sqsubseteq \gamma\left(p^{\sharp}\right)
$$

for all $p^{b} \in P^{b}$ and $p^{\sharp} \in P^{\sharp}$. It is easy to see from (8) and (9) how $\alpha$ and $\gamma$ can be derived from each other. It is not hard to show that $\gamma$ preserves top (i.e., $\gamma\left(T^{\sharp}\right)=T^{b}$ ), is monotonic (i.e., $p^{\sharp} \sqsubseteq q^{\sharp} \Rightarrow \gamma\left(p^{\sharp}\right) \sqsubseteq \gamma\left(q^{\sharp}\right)$ for all $\left.p^{\sharp}, q^{\sharp} \in P^{\sharp}\right)$, and distributes over arbitrary meet (i.e., $\gamma\left(\sqcap Q^{\sharp}\right)=\sqcap\left\{\gamma\left(q^{\sharp}\right) \in P^{b} \mid q^{\sharp} \in Q^{\sharp}\right\}$ for all $\left.Q^{\sharp} \subseteq P^{\sharp}\right)$. Similarly, $\alpha$ preserves bottom, is also monotonic, and distributes over arbitrary join.

Galois Connection from $\mathcal{P}\left(\mathbf{B}^{m}\right)$ to $\mathbf{T}_{\perp}^{m}$. For any $m \in \mathbf{N}$, there is a natural Galois connection $\ll$ from $\mathcal{P}\left(\mathbf{B}^{m}\right)$ to $\mathbf{T}_{\perp}^{m}$, which is most conveniently defined by specifying its concretization function $\gamma: \mathbf{T}_{\perp}^{m} \rightarrow \mathcal{P}\left(\mathbf{B}^{m}\right)$ :

$$
\begin{aligned}
\gamma\left(\left\langle t_{0}, \cdots, t_{m-1}\right\rangle\right) & =\left\{\left\langle b_{0}, \cdots, b_{m-1}\right\rangle \in \mathbf{B}^{m} \mid \forall i<m: t_{i} \neq \mathbf{X} \Rightarrow b_{i}=t_{i}\right\} \\
\gamma(\perp) & =\emptyset
\end{aligned}
$$

for all $\left\langle t_{0}, \cdots, t_{m-1}\right\rangle \in \mathbf{T}^{m}$. In other words, for any ternary vector $t \in \mathbf{T}^{m}, \gamma(t)$ is the set of all boolean vectors $\in \mathbf{B}^{m}$ that agree with $t$ on all non-X components (so $\mathbf{X}$ 's can

3 Our ordering of $\mathbf{T}$ is the reverse of that used in [8,3]. We do so because we want to make clear that the ordering of $\mathbf{T}$ is an abstraction of set inclusion. 
be thought of as "wild cards"). Note that $\gamma$ is in fact a bijection from $\mathbf{T}_{\perp}^{m}$ to those subsets of $\mathbf{B}^{m}$ that are (hyper)cubes. From $\gamma$, the Galois connection $\ll \subseteq \mathcal{P}\left(\mathbf{B}^{m}\right) \times \mathbf{T}_{\perp}^{m}$ and the abstraction function $\alpha: \mathcal{P}\left(\mathbf{B}^{m}\right) \rightarrow \mathbf{T}_{\perp}^{m}$ can be easily derived using (8) and (9). Intuitively, $b \ll t$ iff the cube corresponding to $t$ contains $b$, and $\alpha(b)$ is the element of $\mathbf{T}_{\perp}^{m}$ that corresponds to the smallest cube in $\mathbf{B}^{m}$ that contains $b$.

\section{A.4 Proof of Theorem 1}

We prove the two directions of $\Leftrightarrow$ separately.

The $\Rightarrow$ direction: Suppose this is not true, i.e., $M \models_{\text {Set }} A$ but $M \not \models A$. Then $M \not \models A$ implies that there exist $\tau \in \operatorname{Traj}(M)$ and $\rho \in \operatorname{Runs}(A)$ such that $|\tau|=|\rho|$, $\tau \models \models_{a} \rho$, and $\tau^{\prime} \models_{c} \rho^{\prime}$, but $\operatorname{last}(\tau) \notin \pi_{c}(\operatorname{last}(\rho))$, where $\tau^{\prime}$ and $\rho^{\prime}$ are the prefixes of $\tau$ and $\rho$ respectively such that $\left|\tau^{\prime}\right|=|\tau|-1$ and $\left|\rho^{\prime}\right|=|\rho|-1$. We claim that for all $i \in \mathbf{N}$ with $0 \leq i<|\tau|, \tau[i] \in \Phi_{*}(\rho[i])$. This is proved by induction on $i$. The base case $i=0$ is trivial, since $\Phi_{*}\left(s_{0}\right)=C$. For the induction step, assume the claim is true for $i<|\tau|-1$. Then $\tau[i] \in \Phi_{*}(\rho[i]) \cap \pi_{a}(\rho[i])$, since $\tau=_{a} \rho$. So $\tau[i+1] \in F(\rho[i])\left(\Phi_{*}(\rho[i])\right)$, since $\tau \in \operatorname{Traj}(M)$. But, since $\Phi_{*}=\mathcal{F}\left(\Phi_{*}\right), F(\rho[i])\left(\Phi_{*}(\rho[i])\right) \subseteq \Phi_{*}(\rho[i+1])$. This completes the induction step, so the claim is true. But the claim implies that last $(\tau) \in$ $\Phi_{*}(\operatorname{last}(\rho)) \cap \pi_{a}(\operatorname{last}(\rho))$, which implies $\operatorname{last}(\tau) \in \pi_{c}(\operatorname{last}(\rho))$ because $M \models=_{\text {Set }} A$. So $\operatorname{last}(\tau) \in \pi_{c}(\operatorname{last}(\rho))$ and $\operatorname{last}(\tau) \notin \pi_{c}(\operatorname{last}(\rho))$, a contradiction.

The $\Leftarrow$ direction: Since $F(s)$ is distributive over arbitrary union for all $s \in S$, a well-known result from DFA [4] states that the least fixpoint solution $\Phi_{*}$ of the equation $\Phi=\mathcal{F}(\Phi)$ is identical to the union-over-all-runs solution. More precisely, $\Phi_{*}$ must satisfy the following equation:

$$
\Phi_{*}(s)=\text { if }\left(s=s_{0}\right) \text { then } C \text { else } \cup\left\{G\left(\rho^{\prime}\right) \mid \rho^{\prime} \in \operatorname{Runs}(A) \wedge\left(\operatorname{last}\left(\rho^{\prime}\right), s\right) \in R\right\}
$$

for all $s \in S$, where $G: \operatorname{Runs}(A) \cup\{\langle\rangle\} \rightarrow \mathcal{P}(C)$ is defined inductively by $G(\langle\rangle)=C$ and $G(\rho \frown\langle s\rangle)=F(s)(G(\rho))$. Let $c \in C$ and $s \in S$. Using the definitions of $G, F$, and $M$, the equation above can be rephrased as:

$$
\begin{aligned}
c \in \Phi_{*}(s) \Leftrightarrow \exists \tau & \in \operatorname{Traj}(M): \exists \rho \in \operatorname{Runs}(A): \\
& |\tau|=|\rho| \wedge \operatorname{last}(\tau)=c \wedge \operatorname{last}(\rho)=s \wedge \\
& \forall i \in \mathbf{N}: 0 \leq i<|\tau|-1 \Rightarrow \tau[i] \in \pi_{a}(\rho[i])
\end{aligned}
$$

Conjoining $c \in \pi_{a}(s)$ to both sides, we get:

$$
\begin{aligned}
& c \in \Phi_{*}(s) \cap \pi_{a}(s) \Leftrightarrow \exists \tau \in \operatorname{Traj}(M): \exists \rho \in \operatorname{Runs}(A): \\
& |\tau|=|\rho| \wedge \operatorname{last}(\tau)=c \wedge \operatorname{last}(\rho)=s \wedge \tau \mid=_{a} \rho
\end{aligned}
$$

Now the definition of $M \models A$ shows that the $\Leftarrow$ direction is indeed true.

\section{A.5 Proof of Theorem 2}

Throughout this proof, we will freely use the right half of (9): $p \ll \hat{p} \Leftrightarrow p \subseteq \gamma(\hat{p})$ for all $p \in \mathcal{P}(C)$ and $\hat{p} \in \hat{P}$. For set-theoretic STE, the notations are exactly the same as in Section 2.3 and Appendix A.4, except that the (concrete) trajectory assertion is $\gamma(\hat{A})=\left(S, s_{0}, R, \gamma\left(\hat{\pi}_{a}\right), \gamma\left(\hat{\pi}_{c}\right)\right)$ instead of $A$.

Claim 1: $p \ll \hat{p} \Rightarrow F(s)(p) \ll \hat{F}(s)(\hat{p})$ for all $p \in \mathcal{P}(C), \hat{p} \in \hat{P}$, and $s \in S$. This is proved as follows: 


$$
\begin{aligned}
\gamma(\hat{F}(s)(\hat{p})) & =\gamma\left(\hat{M}\left(\hat{\pi}_{a}(s) \sqcap \hat{p}\right)\right) & & \{\text { definition of } \hat{F}\} \\
& \supseteq M\left(\gamma\left(\hat{\pi}_{a}(s) \sqcap \hat{p}\right)\right) & & \{\ll \text { is a simulation relation }\} \\
& =M\left(\gamma\left(\hat{\pi}_{a}(s)\right) \cap \gamma(\hat{p})\right) & & \{\text { distributivity of } \gamma\} \\
& \supseteq M\left(\gamma\left(\hat{\pi}_{a}(s)\right) \cap p\right) & & \{p \ll \hat{p} \text { and monotonicity of } M\} \\
& =F(s)(p) & & \{\text { definition of } F\}
\end{aligned}
$$

where $\{\cdots\}$ 's give the justifications of proof steps.

Claim 2: $\Phi_{*}(s) \ll \hat{\Phi}_{*}(s)$ for all $s \in S$. Since $\Phi_{*}(s)=\lim \Phi_{n}(s)$ and $\hat{\Phi}_{*}(s)=$ $\lim \hat{\Phi}_{n}(s)$, it suffices to prove that $\Phi_{n}(s) \ll \hat{\Phi}_{n}(s)$ for all $s \in S$ and $n \in \mathbf{N}$. This is proved by induction on $n$. The base case is trivial, since a Galois connection always relates the two bottoms. For the induction step, assume $\Phi_{n}(s) \ll \hat{\Phi}_{n}(s)$ for all $s \in S$. That $\Phi_{n+1}\left(s_{0}\right) \ll \hat{\Phi}_{n+1}\left(s_{0}\right)$ is also trivial, since a Galois connection always relates the two tops. For any $s_{0} \neq s \in S$, we have:

$$
\begin{aligned}
\gamma\left(\hat{\Phi}_{n+1}(s)\right) & =\gamma\left(\sqcup\left\{\hat{F}\left(s^{\prime}\right)\left(\hat{\Phi}_{n}\left(s^{\prime}\right)\right) \mid\left(s^{\prime}, s\right) \in R\right\}\right) & & \{(6)\} \\
& \supseteq \cup\left\{\gamma\left(\hat{F}\left(s^{\prime}\right)\left(\hat{\Phi}_{n}\left(s^{\prime}\right)\right)\right) \mid\left(s^{\prime}, s\right) \in R\right\} & & \{\text { monotonicity of } \gamma\} \\
& \supseteq \cup\left\{F\left(s^{\prime}\right)\left(\Phi_{n}\left(s^{\prime}\right)\right) \mid\left(s^{\prime}, s\right) \in R\right\} & & \left\{\Phi_{n}(s) \ll \hat{\Phi}_{n}(s) \text { and claim } 1\right\} \\
& =\Phi_{n+1}(s) & & \{(5)\}
\end{aligned}
$$

This completes the induction step, so the claim is true.

Finally, suppose $\left.\hat{M}\right|_{\text {Lat }} \hat{A}$. Then, for all $s \in S$, we have:

$$
\begin{aligned}
\gamma\left(\hat{\pi}_{c}(s)\right) & \supseteq \gamma\left(\hat{\Phi}_{*}(s) \sqcap \hat{\pi}_{a}(s)\right) & & \left\{\hat{M} \models_{\text {Lat }} \hat{A}\right. \text { and mon } \\
& =\gamma\left(\hat{\Phi}_{*}(s)\right) \cap \gamma\left(\hat{\pi}_{a}(s)\right) & & \{\text { distributivity of } \gamma\} \\
& \supseteq \Phi_{*}(s) \cap \gamma\left(\hat{\pi}_{a}(s)\right) & & \{\text { claim } 2\}
\end{aligned}
$$

Therefore, $M=_{\text {Set }} \gamma(\hat{A})$.

\section{References}

1. P. Cousot and R. Cousot, "Abstract Interpretation: A Unified Lattice Model for Static Analysis of Programs by Construction or Approximation of Fixpoints", pp.238-252 of Conf. Rec. of 4th ACM Symp. on Principles of Programming Languages (POPL'ry), Oct. 1977.

2. B.A. Davey and H.A. Priestley, Introduction to Lattices and Order, Cambridge University Press, 1990.

3. A. Jain, "Formal Hardware Verification by Symbolic Trajectory Evaluation", Ph.D. Thesis supervised by R.E. Bryant, Carnegie-Mellon University, July 1997.

4. G.A. Kildall, "A Unified Approach to Global Program Optimization", pp.194206 of Conf. Rec. of 1st ACM Symp. on Principles of Programming Languages (POPL'Y3), Oct. 1973.

5. K.L. McMillan, Symbolic Model Checking, Kluwer Academic Publishers, 1993.

6. S.S. Muchnick, Advanced Compiler Design and Implementation, Morgan Kaufmann Publishers, 1997.

7. D.A. Schmidt and B. Steffen, "Data-Flow Analysis as Model Checking of Abstract Interpretations", invited tutorial paper, Proc. 5th Static Analysis Symposium, G. Levi (ed.), Pisa, Sep. 1998, Springer LNCS 1503.

8. C.-J.H. Seger and R.E. Bryant, "Formal Verification by Symbolic Evaluation of Partially-Ordered Trajectories", Formal Methods in System Designs, Vol. 6, No. 2, pp. 147-189, March 1995. 